\title{
Reactions of aromatics in space and connections to the carbon chemistry of Solar System materials
}

\author{
Max Bernstein \\ Astrophysics Branch, NASA/Ames Research Center \\ email: Max.Bernstein@nasa.gov
}

\begin{abstract}
Polycyclic aromatic hydrocarbons (PAHs) and related aromatic materials are thought to be the most abudant class of organic carbon in the universe, being present in virtually all phases of the ISM, and abundant in carbonaceous meteorites and asteroid and comet dust. The basic PAH skeleton is proposed to have formed in outflows of carbon rich stars, and isotopic measurements of extraterrestrial graphitic carbon is consistent with this notion. However, functionalized aromatics bearing oxygen atoms, aliphatic domains, and deuterium enrichments have been extracted from meteorites and more recently been measured in IDPs and Stardust retuned comet samples. Exposure of remnant circumstellar PAHs to energetic processing at low temperature in the presense of $\mathrm{H}_{2} \mathrm{O}$ is the most parsimonious explanation for these observations.

We will present laboratory infrared spectra of various aromatic species and PAH cations in solid $\mathrm{H}_{2} \mathrm{O}$ under conditions relevant for comparsion to absorptions attributed to PAHs observed towards objects embedded in dense clouds. In addition, we shall describe the reactions of PAHs under these conditions in the lab when they are exposed to energetic processing. Finally, we will propose a mechanism, and make specific predictions regarding the structures and distribution of deuterium that should be observed in extraterrestrial samples if low temperature ice radiation chemistry is playing a role in the formation of the molecules seen in Solar System materials.
\end{abstract}

\section{Discussion}

CoDY: Did you ever see evidence in your quinoline experiments of nitrogen elimination? It's intriguing to think that if you are hydrogenating right next to the nitrogen, you might actually have some products where you've lost nitrogen.

BERnSTEIN: It's possible that it might have happened and that we did not see it. We tried to keep the doses extremely low to keep the chemistry fairly simple and to avoid blowing these things apart. I think quinoline (a naphthalene with a single nitrogen in one of its rings) is fairly stable. Preliminary indications are that compounds that have more than one nitrogen in them break apart much more easily. I think we were doing these experiments under conditions where we wouldn't have seen much nitrogen elimination.

KHARE: Your experiments are done at a very low temperature. At that temperature the products that you make won't fly away from the window. The only things that might escape will be things like $\mathrm{N}_{2}, \mathrm{CH}_{4}$, and $\mathrm{CO}$.

Berstein: Yes. In fact, not even them. We generally do these experiments on a salt window that is cooled to $\sim 10 \mathrm{~K}$. At this temperature the only thing that escapes from the ice is $\mathrm{H}_{2}$, which you generate during the course of photolysis. If you do these experiments at temperatures above which some of the ice components, say methane, can sublime, then it will be harder to add that methyl group. The reaction efficiencies depend 
to some extent on the residence time of the associated molecules in the ice. If you are interested in reaction between a $\mathrm{PAH}$ and $\mathrm{H}_{2} \mathrm{O}$, it doesn't matter so much. If you are specifically interested in the reaction between a PAH and methane, then it would be more temperature dependent. However, the reactions would not change at all if you were to do them at lower temperatures.

SANDFORD: I am going to disagree on one point with both of you. When you photolyze these ices at these low temperatures, the photons break some of the molecules apart to produce ions and radicals. Very few of these are actually on the surface of the ice. The reason they don't escape is because they are trapped in the bulk of the solid $\mathrm{H}_{2} \mathrm{O}$ rich ice, not because the temperature is below the sublimation temperature of all the photoproducts. A lot of the group addition happens when the ice is warmed and its components become mobile as the $\mathrm{H}_{2} \mathrm{O}$ sublimes away. So, in fact, at any temperature below the sublimation point of $\mathrm{H}_{2} \mathrm{O}$, you will get many of the very same products because you are just using the $\mathrm{H}_{2} \mathrm{O}$ to store ions and radicals until they react during the warm up.

Bernstein: That is true. If you were to vapor deposit a water:methane mixture at a low enough temperature that the methane is trapped, it would still be available for reactions even if the ice is later warmed.

KOBAYASHI: I understand that there are many aromatic compounds in space. Do you have any idea why aromatic amino acids are rarely found in meteorites or seen in other environments in space?

BERnstein: My intuition, based on work we published in Nature in 2002 on the formation of amino acids, suggests that it's just easier to make simpler molecules. Using fairly simple starting materials, the kinds of molecules that are common in space, one certainly makes more glycine than anything else. The production of alanine or serine, where you are just adding one more carbon atom, shows considerably diminished yields. I imagine that we could make a naphthyl amino acid, but I think the more complicated the product is, the harder it is to make. The full answer is unlikely to be simple, however, since there are obviously multiple mechanisms leading to the amino acids that are seen in meteorites.

HenNING: You start with the assumption that PAHs in the diffuse interstellar medium have no side groups. Why do you make this assumption?

BERnsteIn: I make that assumption for two reasons. First, we've heard the basic idea that in C-rich stellar outflows all the oxygen get used up to make carbon monoxide and any remaining carbon forms some sort of sooty material that contains just carbon and hydrogen. In this simple picture there's no $\mathrm{O}$ available for O-containing side groups. I am also presuming that side groups are going to be less stable against loss by photolysis in the diffuse ISM. It's possible that some functional groups could be present, but I'm simply starting with the worst-case scenario. I want to answer the question, why is there this richness in the chemistry of the aromatic compounds in meteorites? If I start with the simplest case, an aromatic molecule that has nothing on it, then it's not activated in any way. It's the toughest thing to drive in the chemistry. If I can get the process to work in that worst case scenario, then it should be even easier if you tell me that I get to start with methyl groups or bridging $\mathrm{CH}_{2}$ groups.

HenNing: But then the process will be different, won't it? 
BeRnstein: Yes, possibly, and then you get to make specific predictions. If we could get to the point where we could examine the hot spots in Stardust samples or in IDPs and say this or that functional group carries an isotopic enrichment, we could answer the question of whether they are circumstellar or whether they were introduced at another point. If there are clear correlations between certain functional groups and isotopic anomalies we could learn a great deal.

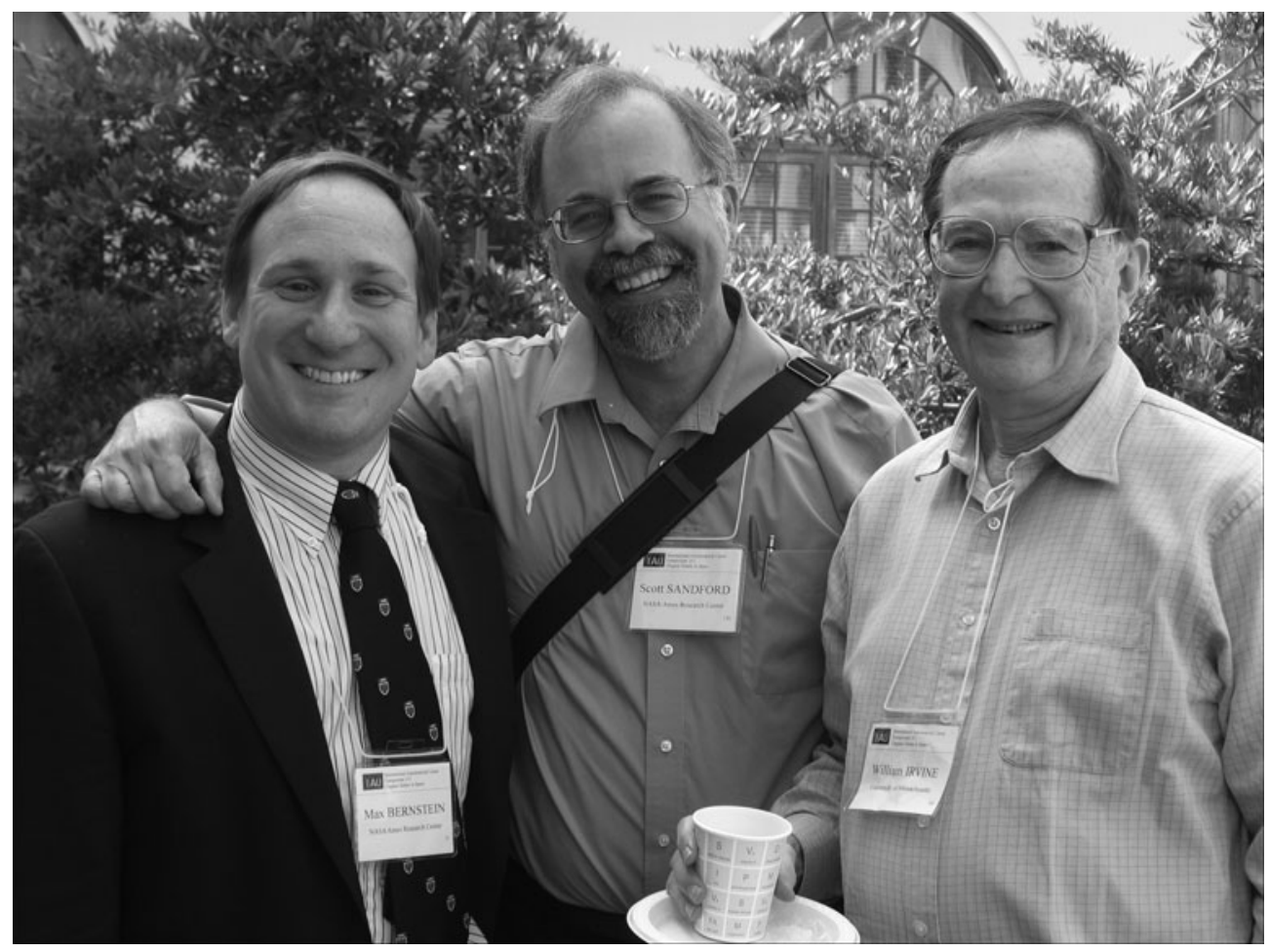

From left to right: Max Bernstein, Scott Sandford, and William Irvine (photo by Dale Cruikshank). 


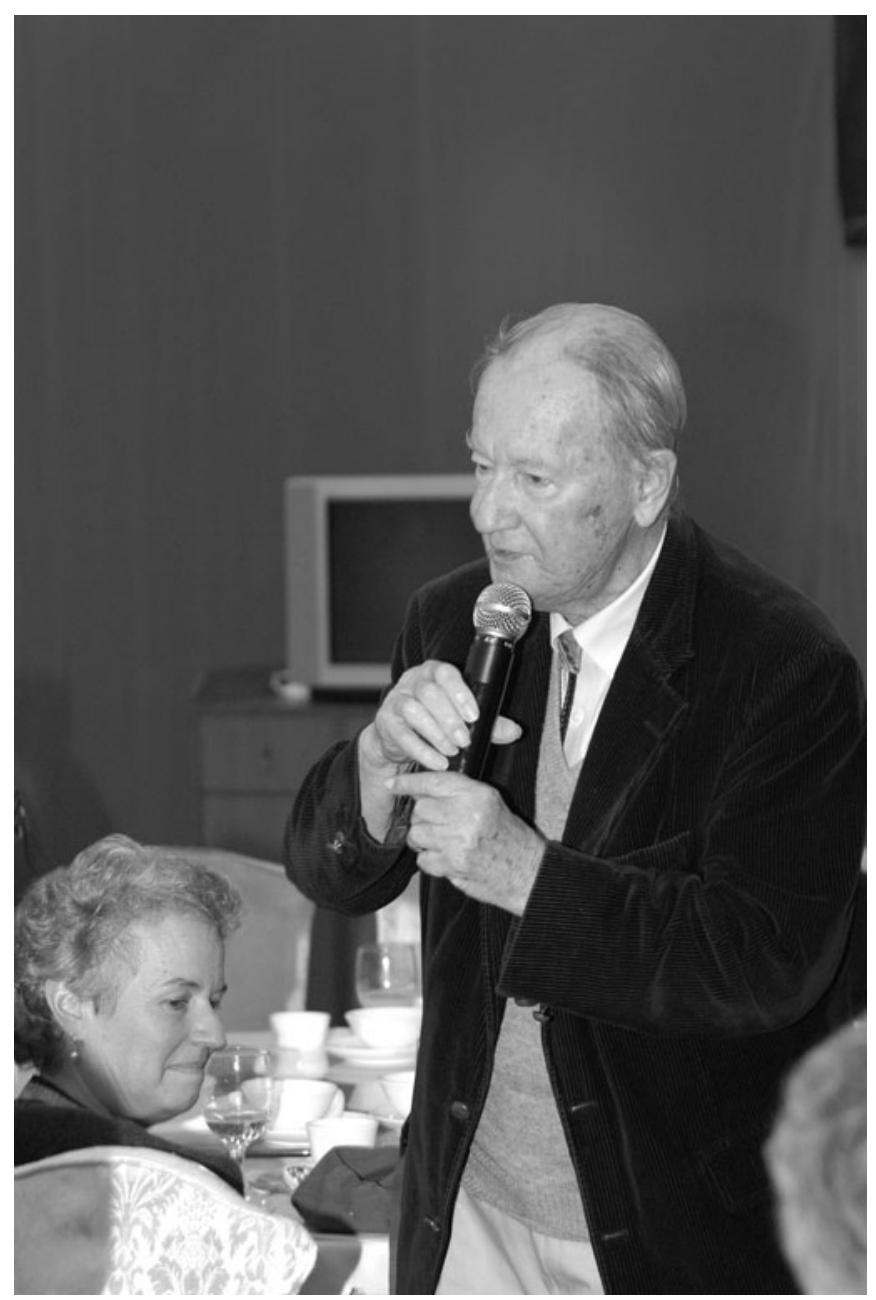

Cliff Matthews singing during his after-dinner speech at the banquet. 